\title{
Metamagnetic phase transition in ferromagnetic superconductor URhGe
}

\author{
V.P.Mineev ${ }^{1,2^{*}}$ \\ ${ }^{1}$ Universite Grenoble Alpes, CEA, IRIG, PHELIQS, F-38000 Grenoble, France \\ ${ }^{2}$ Landau Institute for Theoretical Physics, 142432 Chernogolovka, Russia
}

(Dated: March 29, 2021)

\begin{abstract}
Ferromagnetic superconductor URhGe has orthorhombic structure and possesses spontaneous magnetisation along the c-axis. Magnetic field directed along the $b$-axis suppresses ferromagnetism in $c$-direction and leads to a metamagnetic transition into polarised paramagnetic state in the $b$ direction. The theory of these phenomena based on the specific magnetic anisotropy of this material in $(b, c)$ plane is given. Line of the first order metamagnetic transition ends at a critical point. The Van der Waals - type description of behaviour of physical properties near this point is developed. The triplet superconducting state destroyed by orbital effect is recreated in vicinity of the transition. It is shown that the reentrance of superconductivity is caused by the sharp increase of magnetic susceptibility in $b$ direction near the metamagnetic transition. The specific behaviour of the upper critical field in direction of spontaneous magnetisation in UCoGe and in $\mathrm{UGe}_{2}$ related to the field dependence of magnetic susceptibility is discussed.
\end{abstract}

\section{INTRODUCTION}

Investigations of uranium superconducting ferromagnets $\mathrm{UGe}_{2}$, URhGe and UCoGe continue attract attention mostly due to the quite unusual nature of its superconducting states created by the magnetic fluctuations (see the recent experimental [1] and theoretical [2] reviews and references therein). They have orthorhombic crystal structure and the anisotropic magnetic properties. The spontaneous magnetisation is directed along $a$ axis in $\mathrm{UGe}_{2}$ and along $c$-axis in URhGe and UCoGe. The ferromagnetic state in the two last materials is suppressed by the external magnetic field $H_{y}$ directed along $b$ crystallographic direction. In URhGe at field $H_{y}=H_{c r} \approx 12$ $\mathrm{T}$ the second order phase transition to ferromagnetic state is transformed to the transition of the first order [3]. The superconducting state suppressed [4] in much smaller fields $H_{y} \approx 2 \mathrm{~T}$ is reappeared in vicinity of the first order transition in field interval $(9,13) \mathrm{T}$. The phenomenological theory of this phenomenon has been developed in Ref.5 (see also [2]). According to this theory the state arising in fields above the suppression of spontaneous magnetisation in $c$-direction is the paramagnetic state.

There was established, however, [3, 6, 7] that in fields above $H_{c r}$ the magnetisation along $b$ direction looks like it has field independent "spontaneous" component

$$
M_{y}=M_{y 0}+\chi_{y} H_{y}
$$

This state is called polarised paramagnetic state. The formation of this state is related with so called metamagnetic transition observed in several heavy-fermion compounds (see the paper [8] and the more recent publication [9] and references therein). To take into account the formation of polarised paramagnetic state one must intro-

\footnotetext{
*E-mail: vladimir.mineev@cea.fr
}

duce definite modifications in the treatment performed in [5]. Here I present the corresponding derivation.

The paper is organized as follows. In the Section II after the brief reminder of results of the paper [5] the description of the metamagnetic transition is presented. It is based on the specific phenomenon of magnetic anisotropy in URhGe obtained with a local spindensity approximation calculations by Alexander Shick [10. After the general consideration of the metamagnetic transition the modifications introduced by the uniaxial stress are considered. Then the Van der Waals type theory of phenomena near the metamagnetic critical point is developed and some physical properties are discussed.

The phenomenon of the reentrant superconducting state is explained in the Section III. It is shown that the recreation of superconductivity is caused by the sharp increase in the magnetic susceptibility [7] in $b$ direction near the metamagnetic transition. This Section also contains the qualitative description of the specific behaviour of the upper critical field in direction of spontaneous magnetisation in $\mathrm{UCoGe}$ and in $\mathrm{UGe}_{2}$ related to the field dependence of magnetic susceptibility.

The Conclusion contains the summary of the results.

\section{METAMAGNETIC TRANSITION IN URhGe}

As in the previous publications ([2, 5]) I shall use $x, y, z$ as the coordinates pinned to the corresponding crystallographic directions $a, b, c$. The Landau free energy of an orthorhombic ferromagnet in magnetic field $\mathbf{H}(\mathbf{r})=H_{y} \hat{y}$ is

$$
\begin{gathered}
F=\alpha_{z} M_{z}^{2}+\beta_{z} M_{z}^{4}+\delta_{z} M_{z}^{6} \\
+\alpha_{y} M_{y}^{2}+\beta_{y} M_{y}^{4}+\delta_{y} M_{y}^{6}+\beta_{y z} M_{z}^{2} M_{y}^{2}-H_{y} M_{y}
\end{gathered}
$$

Here

$$
\alpha_{z}=\alpha_{z 0}\left(T-T_{c 0}^{c}\right), \quad \alpha_{y}>0
$$


and I bear in mind the terms of the sixth order in powers of $M_{z}, M_{y}$ and also the fact that in the absence of a field in $x$-direction the magnetisation along hard $x$-direction $M_{x}=0$.

\section{A. Transition ferro-para}

Let us remind first the treatment developed in Ref.5 undertaken in the assumption $\beta_{y}>0$. Then in constant magnetic field $\mathbf{H}=H_{y} \hat{y}$ the equilibrium magnetisation projection along the $y$ direction

$$
M_{y} \approx \frac{H_{y}}{2\left(\alpha_{y}+\beta_{y z} M_{z}^{2}\right)}
$$

is obtained by minimisation of free energy 2 in respect of $M_{y}$ neglecting the higher order terms. Substituting this expression back to 2 we obtain

$$
F=\alpha_{z} M_{z}^{2}+\beta_{z} M_{z}^{4}+\delta_{z} M_{z}^{6}-\frac{1}{4} \frac{H_{y}^{2}}{\alpha_{y}+\beta_{y z} M_{z}^{2}},
$$

that gives after expansion of the denominator in the last term,

$$
F=-\frac{H_{y}^{2}}{4 \alpha_{y}}+\tilde{\alpha}_{z} M_{z}^{2}+\tilde{\beta}_{z} M_{z}^{4}+\tilde{\delta}_{z} M_{z}^{6}+\ldots
$$

where

$$
\begin{gathered}
\tilde{\alpha}_{z}=\alpha_{z 0}\left(T-T_{c 0}\right)+\frac{\beta_{y z} H_{y}^{2}}{4 \alpha_{y}^{2}}, \\
\tilde{\beta}_{z}=\beta_{z}-\frac{\beta_{y z}}{\alpha_{y}} \frac{\beta_{y z} H_{y}^{2}}{4 \alpha_{y}^{2}}, \\
\tilde{\delta}_{z}=\delta_{z}+\frac{\beta_{y z}^{2}}{\alpha_{y}^{2}} \frac{\beta_{y z} H_{y}^{2}}{4 \alpha_{y}^{2}} .
\end{gathered}
$$

Thus, in a magnetic field perpendicular to the direction of spontaneous magnetization the Curie temperature decreases as

$$
T_{c}=T_{c}\left(H_{y}\right)=T_{c 0}-\frac{\beta_{y z} H_{y}^{2}}{4 \alpha_{y}^{2} \alpha_{z 0}} .
$$

The coefficient $\tilde{\beta}_{z}$ also decreases with $H_{y}$ and reaches zero at

$$
H_{y}=H^{\star}=\frac{2 \alpha_{y}^{3 / 2} \beta_{z}^{1 / 2}}{\beta_{y z}} .
$$

At this field under fulfilment the condition,

$$
\frac{\alpha_{z 0} \beta_{y z} T_{c 0}}{\alpha_{y} \beta_{z}}>1
$$

the Curie temperature (10) is still positive and the phase transition from the ferromagnetic to the paramagnetic state becomes the transition of the first order (Fig 1a). The point $\left(H^{\star}, T_{c}\left(H^{\star}\right)\right)$ on the line paramagnetferromagnet phase transition is a tricritical point. The qualitative field dependences of the normalised Curie temperature $t_{c}\left(H_{y}\right)=\frac{T_{c}\left(H_{y}\right)}{T_{c 0}}$ and $b\left(H_{y}\right)=\frac{\tilde{\beta}_{z}}{\beta_{z}}$ are plotted in Fig 1a.

On the line of the first order phase transition from the ferromagnet to the paramagnet state the $M_{z}$ component of magnetisation drops from $M_{z}^{\star}$ to zero [2]. The $M_{y}$ component jumps from $M_{y} \approx \frac{H^{\star}}{2\left(\alpha_{y}+\beta_{y z} M_{z}^{\star 2}\right)}$ to $M_{y} \approx \frac{H^{\star}}{2 \alpha_{y}}$. Then at fields $H_{y}>H^{\star}$

$$
M_{y} \approx \frac{H_{y}}{2 \alpha_{y}}
$$

proportional to the external field. This contradicts experimental observations [3, 6, 7] which demonstrate the presence of a "spontaneous" part of magnetization in the field above the transition in accordance with Eq.(1).

\section{B. Transition ferro-polarised para}

The part of free energy depending on $M_{y}$

$$
F_{y}=\alpha_{y} M_{y}^{2}+\beta_{y} M_{y}^{4}+\delta_{y} M_{y}^{6}+\beta_{y z} M_{z}^{2} M_{y}^{2}-H_{y} M_{y}
$$

can be used also far from the transition to the ferromagnetic state in the temperature region where $M_{z}$ is not small. The important fact obtained with the local spindensity approximation calculations [10] is that the coefficient $\beta_{y}<0$. In frame of isotropic Fermi liquid model the negativeness of the fourth order term in the expansion of the free energy in power of magnetic moment is usually ascribed to the peculiar behaviour of the electron density of states (see the review [11] and referencies therein). In the orthorhombic URhGe this specific magnetocrystalline anisotropy reveals itself in the system of magnetic moments localised on the uranium atoms [12].

The $M_{y}$ component of magnetisation is determined by the equation

$$
2 \tilde{\alpha}_{y} M_{y}+4 \beta_{y} M_{y}^{3}+6 \delta_{y} M_{y}^{5}=H_{y},
$$

where

$$
\tilde{\alpha}_{y}=\alpha_{y}+\beta_{y z} M_{z}^{2}
$$

Taking into account the third order term we obtain

$$
M_{y} \approx \frac{H_{y}}{2 \tilde{\alpha}_{y}}-\frac{\beta_{y} H_{y}^{3}}{2 \tilde{\alpha}_{y}^{4}} .
$$

The coefficient $\beta_{y}<0$ and we see that the increase of magnetisation occurs faster than it was according to Eq. (4).

The shape of $M_{y}\left(H_{y}\right)$ depends from the temperature and pressure dependence of coefficients $\alpha_{y}, \beta_{y}, \delta_{y}$. In particular, the coefficient $\tilde{\alpha}_{y}(T)$ is decreasing function of temperature and at temperature decrease the field dependence of $M_{y}$ transfers from the monotonous growth taking place at $\beta_{y}^{2}<\frac{5}{3} \tilde{\alpha}_{y} \delta_{y}$ to the S-shape dependence 
realising at $\beta_{y}^{2}>\frac{5}{3} \tilde{\alpha}_{y} \delta_{y}$. This transformation occurs at some temperature $T_{c r}$ such that in the dependence $H_{y}\left(M_{y}\right)$ appears an inflection point. It is determined by the equations

$$
\frac{\partial H_{y}}{\partial M_{y}}=0, \quad \frac{\partial^{2} H_{y}}{\partial M_{y}^{2}}=0
$$

having common solution

$$
M_{c r}^{2}=-\frac{\beta_{y}}{5 \delta_{y}},
$$

at $\beta_{y}^{2}=\frac{5}{3} \tilde{\alpha}_{y} \delta_{y}$. The corresponding critical field is

$$
H_{c r}=H_{y}\left(M_{c r}\right)=\frac{16}{5 \sqrt{3}} \frac{\tilde{\alpha}_{y}^{3 / 2}}{\left|\beta_{y}\right|^{1 / 2}} .
$$

At $T<T_{c r}$ the inequality

$$
\beta_{y}^{2}>\frac{5}{3} \tilde{\alpha}_{y} \delta_{y}
$$

is realised and the equation $\frac{\partial H_{y}}{\partial M_{y}}=0$ acquires two real solutions, hence, the field dependence of $M_{y}$ acquires the S-shape plotted at Fig.1b. Equilibrium transition from the lower to the upper part of the curve $M_{y}\left(H_{y}\right)$ corresponds to a vertical line connecting the points $M_{1}$ and $M_{2}$ defined by the Maxwell rule $\int_{1}^{2} M(H) d H=0$. The integration is performed along the curve $M_{y}\left(H_{y}\right)$. The $M_{y}$ component of magnetisation jumps from $M_{1}$ to $M_{2}$ (see Fig1b).

At temperatures above $T_{c r}$ the jump transforms into the crossover which is the temperature-field region characterised by the fast growth $M_{y}$. The lower boundary of this region roughly coincides with the Curie temperature (see Fig2.). The Curie temperature decreasing with growth of magnetisation $M_{y}$

$$
T_{c}\left(H_{y}\right)=T_{c 0}-\frac{\beta_{y z} M_{y}^{2}}{\alpha_{z 0}}
$$

falls down to zero or even to negative value at sharp increase of $M_{y}$ in vicinity of the critical field $H_{c r}$ and the ferromagnetic order along $z$-direction disappears. Thus, at $T<T_{c r}$ and $H_{y}=H_{c r}$ we have the phase transition of the first order from the ferromagnetic state with spontaneous magnetisation along $z$-direction to the polarised paramagnetic state with induced magnetisation along $y$ direction (Fig2).

The described jump-like transition is realised in the cylindrical specimen in the magnetic field parallel to the cylinder axis. In specimens of the arbitrary shape with demagnetisation factor $n$ the transition occurs in some field interval where the specimen is filled by the domains with different magnetisation.

When the critical field $H_{c r}$ is smaller than the critical field of transition ferro-para $H^{\star}$, the ferro-para transition, discussed in the previous section does not occurs.

At $T<T_{c r}$ in fields $H_{y}$ exceeding $H_{c r}$ the field dependence of $M_{y}$ component of magnetisation behaves in accordance with Eq.(1) corresponding to the experimental observations.

\section{Uniaxial stress effects}

It is known that a hydrostatic pressure applied to URhGe crystals stimulate ferromagnetism and at the same time suppresses the superconducting state 13 and the reentrant superconducting state [14] as well. The later is also shifted to a bit higher field interval. On the contrary, the uniaxial stress along $b$-direction suppresses the ferromagnetism decreasing the Curie temperature and stimulates the superconducting state so strongly that it leads to the coalescence of the superconducting and reentrant superconducting regions in the $\left(H_{y}, T\right)$ phase diagram [15]. The phenomelogical description of these phenomena was undertaken in the paper [16]. There was shown that both coefficients $\alpha_{z}$ and $\alpha_{y}$ in the Landau free energy Eq.(2) acquire the linear uniaxial pressure dependence

$$
\begin{gathered}
\alpha_{z}\left(P_{y}\right)=\alpha_{z 0}\left(T-T_{c 0}\right)+A_{z} P_{y}, \\
\alpha_{y}\left(P_{y}\right)=\alpha_{y}-\left|A_{y}\right| P_{y}
\end{gathered}
$$

corresponding to the moderate uniaxial pressure suppression of the Curie temperature

$$
T_{c}\left(P_{y}\right)=T_{c 0}-\frac{A_{z} P_{y}}{\alpha_{z 0}},
$$

reported in [15] in the absence of an external field. However, under the external field along $y$-direction the drop of the Curie temperature Eq.10 is accelerated

$$
\left.T_{c}\left(H_{y}, P_{y}\right)\right) \approx T_{c 0}-\frac{A_{z} P_{y}}{\alpha_{z 0}}-\frac{\beta_{y z} H_{y}^{2}}{4\left(\alpha_{y}\left(P_{y}\right)\right)^{2} \alpha_{z 0}}
$$

in correspondence with the observed behaviour. Moreover, the uniaxial stress causes strong decrease of the critical field Eq. 20

$$
H_{c r}=H_{y}\left(M_{c r}\right)=\frac{16}{5 \sqrt{3}} \frac{\left(\tilde{\alpha}_{y}\left(P_{y}\right)\right)^{3 / 2}}{\left|\beta_{y}\right|^{1 / 2}} .
$$

\section{Van der Waals-type theory near the critical point}

The critical end point temperature for the first order transition in URhGe is $T_{c r}=4 \mathrm{~K}$ and the critical hield is $H_{c r}=12 T$. Let us expand the function $H_{y}\left(M_{y}\right)$ at temperature slightly deviating from critical temperature $T=T_{c r}+t$ and the magnetisation near its critical value $M_{y}=M_{c r}+m$. We have

$$
\begin{aligned}
h=H_{y} & -H_{c r}=b t+\left[\left.\frac{\partial H_{y}}{\partial M_{y}}\right|_{t=0}+2 a t\right] m \\
& +\left.\frac{1}{2} \frac{\partial^{2} H_{y}}{\partial M_{y}^{2}}\right|_{t=0} m^{2}+\left.\frac{1}{6} \frac{\partial^{3} H_{y}}{\partial M_{y}^{3}}\right|_{t=0} m^{3},
\end{aligned}
$$

Here, we neglected by the temperature dependence of the second and the third order terms. Taking into account 


$$
\begin{array}{r}
\text { that }\left.\frac{\partial H_{y}}{\partial M_{y}}\right|_{t=0}=\left.\frac{\partial^{2} H_{y}}{\partial M_{y}^{2}}\right|_{t=0}=0 \text { we obtain } \\
h=b t+2 a t m+4 B m^{3},
\end{array}
$$

which obviously corresponds to the expansion of pressure $p=P-P_{c r}$ in powers of density $\eta=n-n_{c r}$ near the Van der Waals critical point [17].

At $t<0$ according to the Maxwell rule the magnetisation densities of two phases in equilibrium with each other are:

$$
m_{2}=-m_{1}=\sqrt{\frac{-a t}{2 B}} .
$$

The line of phase equilibrium between the two phases below and above the transition is given by the equation

$$
h=b t, t<0 \text {. }
$$

\section{Specific heat}

The specific heat at fixed external field(see [17]) is

$$
C_{h} \propto T \frac{\left(\frac{\partial h}{\partial t}\right)_{m}^{2}}{\left(\frac{\partial h}{\partial m}\right)_{t}}
$$

Then, using Eq. 29 we obtain

$$
C_{h} \propto \frac{b^{2} T}{2 a t+12 B m^{2}} .
$$

Thus, the contribution to heat capacity according to the equation of state 29 near the critical point grows so long $m^{2}$ decreases till to $m_{1}^{2}$ and then begins to fall when $m^{2}$ increases starting from $m_{2}^{2}$ (see Fig3). This is the contribution to the specific heat of the whole system and cannot be directly attributed to the specific heat of itinerant electrons proportional to the electron effective mass.

The low temperature behaviour of the URhGe specific heat in magnetic field has not been established by a direct measurement but was derived [6] by the application of the Maxwell relation $\left(\frac{\partial S}{\partial H_{y}}\right)_{T}=\left(\frac{\partial M_{y}}{\partial T}\right)_{H_{y}}$ from the temperature dependence of the magnetisation $M_{y}\left(T, H_{y}\right)$ in the fixed field. The changes of the ratio $C(T) / T$ have been ascribed to the the electron effective mass dependence from magnetic field [6, 18. This was done in the assumption that URhGe is a weak itinerant ferromagnet, in other words, all the low temperature degrees of freedom in this material belong to the itinerant electron subsystem. As we already mentioned above, the strong magnetic anisotropy of this material [10] points on the importance of the magnetic degrees of freedom localised on the uranium ions and related with crystal field levels [2, 12].

\section{Resistivity}

The magnetic field dependence of effective mass was also found [18, 19] by the aplication the Kadowaki-Woods relation $A\left(H_{y}\right) \propto\left(m^{\star}\right)^{2}$ where coefficient $A$ is a prefactor in the low-temperature dependence of resistivity $\rho=\rho_{0}+A T^{2}$.

The $A\left(H_{y}\right)$ behaviour is determined by the processes of inelastic electron-electron scattering which in the multiband metals interfere with scattering on impurities (see fi 20-24]) and on magnetic excitations with field dependent spectrum. The non-spherical shape of the Fermi surface sheets and the screening of el-el Coulomb interaction can introduce deviations from $T^{2}$ resistivity dependence. So, the physical meaning of the coefficient $A\left(H_{y}\right)$ behaviour is not so transparent and its relationship with the electron effective mass is questionable.

One can also note, that the temperature fit of the experimental data was done in very narrow temperature interval and the $T^{2}$ temperature dependence claimed in 19] seems somewhat unreliable. Compare with the results reported in [13, 25].

\section{Correlation function}

The correlation function of fluctuations of the magnetisation density $m$ near the critical point at $t<0$ behaves similar to the specific heat 17 .

$$
\varphi(\mathbf{k})=\frac{T}{2\left(a t+6 B m^{2}+\gamma_{i j} k_{i} k_{j}\right)} .
$$

This is in correspondence with a marked increase of the NMR relaxation rate $1 / T_{2}$ with field $H_{y}$ increasing toward $12 \mathrm{~T}$ reported in [26, 27].

\section{PHASE TRANSITION TO SUPERCONDUCTING STATE}

The superconducting state in URhGe is completely suppressed by the magnetic field $H_{c 2}(T=0) \approx 2 \mathrm{~T}$ in $y$-direction due to the orbital depairing effect. Then superconductivity recovers in the field interval $9-13 \mathrm{~T}$ around the critical field $H_{c r} \approx 12 \mathrm{~T}$ of the transition of the first order from the ferromagnetic state with spontaneous magnetization along z-direction to the state with induced magnetization along $y$-direction. Evidently such type behaviour is possible if the magnetic field somehow stimulates the pairing interaction surmounting the orbital depairing effect.

In numerous publications starting from the paper by A.Miyake et al [18] the treatment of this phenomenon was related with the assumption of an enhancement of electron effective mass $m^{\star}=m(1+\lambda)$ leading to the enhancement of pairing interaction and consequently of 
the temperature of transition to superconducting state according to the Mc-Millan-like formula [28]

$$
T_{s c} \approx \epsilon \exp \left(-\frac{1+\lambda}{\lambda}\right)
$$

derived in the paper [29] for the superconducting state with $p$-pairing in an itinerant isotropic ferromagnetic metal. Similar to the liquid He-3 in this model there are two independent phase transition to the superconducting state in the subsystems with spin-up and spin-down electrons. The constant $\lambda$ determined by the Hubbard four-fermion interaction [29, 30, increases as we approach but not too much close to ferromagnetic instability. In frame of this model the question of why the growth of the magnetic field $H_{y}$ approaches the ferromagnetic transition remains unanswered.

The following development of this type approach has been undertaken by Yu.Sherkunov and co-authors 31. The reentrant superconductivity and mass enhancement have been associated with the Lifshitz transition 32 which occurs in one of the bands in a finite magnetic field stimulating the splitting of spin-up and spin-down bands. There was established modest enhancement of the transition critical temperature in the field about $10 \mathrm{~T}$. Thus, the model can claim to the qualitative explanation of the superconducting state reentrance. However, it should be noted that the measured [32] quasiparticle mass in the corresponding band does not increase but decreases and remains finite, implying that the Fermi velocity vanishes due to the collapse of the Fermi wave vector. The crosssection of the Fermi surface of this band corresponds to $7 \%$ of the Brillouin zone area. Thus, the reentrance of superconductivity is hardly could be associated with the observed Lifshitz transition.

The models [29, 31] describe the physics of pure itinerant electron subsystem. Such a treatment is approved in application to the ${ }^{3} \mathrm{He}$ Fermi-liquid. The measurements by x-ray magnetic circular dichroism [12] point to the local nature of the URhGe ferromagnetism. Namely, the comparison of the total uranium moment $\mu_{\text {tot }}^{U}$ to the total magnetisation $M_{t o t}$ at different magnitude and direction of magnetic field indicates that the uranium ions dominate the magnetism of URhGe. The same is true also in the parent compound UCoGe 33. So, the magnetic susceptibility $\chi_{i j}(\mathbf{q}, \omega)$ is mostly determined by the localised moments subsystem. Hence, an approach based on the exchange interaction between conduction electrons and magnetic moments localised on uranium atoms seems more appropriate. This type theory has been developed in the paper by Hattori and Tsunetsugu 34. Here, there will be undertaken an other approach allowing explicitly take into account the enhancement of magnetic susceptibility near the metamagnetic transition from the ferromagnet state with spontaneous magnetisation along the $c$-axis to the magnetic state polarised along the $b$-axis.

Using the standard functional-integral representation of the partition function of the system (see fi [35]), we obtain the following term in the fermionic action describing an effective two-particle interaction between electrons:

$$
S_{\text {int }}=-\frac{1}{2} I^{2} \int d x d x^{\prime} S_{i}(x) \mathcal{D}_{i j}\left(x-x^{\prime}\right) S_{j}\left(x^{\prime}\right),
$$

where $\mathbf{S}(\mathbf{r})=\psi_{\alpha}^{\dagger}(\mathbf{r}) \boldsymbol{\sigma}_{\alpha \beta} \psi_{\beta}(\mathbf{r})$ is the operator of the electron spin density, $x=(\mathbf{r}, \tau)$ is a shorthand notation for the coordinates in real space and the Matsubara time, $\int d x(\ldots)=\int d \underline{\mathrm{r}} \int_{0}^{\beta} d \tau(\ldots), I$ is the exchange constant of interaction of itinerant electrons with localised magnetic moments, $\mathcal{D}_{i j}\left(x-x^{\prime}\right)$ is the spin-fluctuation propagator expressed in terms of the dynamical spin susceptibility $\chi_{i j}(\mathbf{q}, \omega)$.

Making use the interaction (36) one can calculate the electron self energy and find the dependence of the electron effective mass from magnetic field as well the temperature of transition to the superconducting state with triplet pairing. The energy of electronic excitations in temperature region where the superconducting state is realised is much smaller than typical energy of magnetic excitations. Hence, in calculation of the superconducting properties one can neglect the frequency dependence of susceptibility.

\section{A. Upper critical field parallel to $c$-axis in UCoGe}

In application to UCoGe in magnetic field parallel to direction of spontaneous magnetisation this program has been accomplished in the paper [36]. There has been considered transition into the equal-spin pairing superconducting state in two-band (spin-up, spin-down) orthorhombic ferromagnetic metal. According to this paper in the simplified case of a single-band (say spin-up) equal-spin pairing superconducting state the critical temperature without including the orbital effect of the field is

$$
T_{s c}=\epsilon \exp \left(-\frac{1+\lambda}{\left\langle N_{0}(\mathbf{k}) \chi_{z z}^{u}\right\rangle I^{2}}\right),
$$

where, as in the McMillan formula, $1+\lambda$ corresponds to the effective mass renormalisation, whereas the pairing amplitude expressed through the odd in momentum part of static susceptibility

$$
\chi_{z z}^{u}=\frac{1}{2}\left[\chi_{z z}\left(\mathbf{k}-\mathbf{k}^{\prime}\right)-\chi_{z z}\left(\mathbf{k}+\mathbf{k}^{\prime}\right)\right]
$$

which is the main source of the critical temperature dependence from magnetic field. Here,

$$
\chi_{z z}(\mathbf{k})=\frac{1}{\chi_{z}^{-1}+2 \gamma_{i j} k_{i} k_{j}},
$$

and $\chi_{z}=\chi_{z}\left(H_{z}\right)$ is the $z$-component of susceptibility in the finite field $H_{z}$. Its magnitude at $H_{z} \rightarrow 0$ we will denote $\chi_{z 0}$. The angular brackets denote averaging over the Fermi surface and $N_{0}(\mathbf{k})$ is the angular dependent 
density of electronic states on the Fermi surface,

$$
\left\langle N_{0}(\mathbf{k}) \chi_{z z}^{u}\left(H_{z}\right)\right\rangle \approx \frac{2\left\langle N_{0}(\mathbf{k}) \hat{k}_{z}^{2}\right\rangle k_{F}^{2} \chi_{z}}{\left(2 \chi_{z}\right)^{-1}+4 \gamma_{z z} k_{F}^{2}} .
$$

The denominator in the exponent of Eq.37) can be expressed through its value at $H_{z} \rightarrow 0$

$$
\frac{\left\langle N_{0}(\mathbf{k}) \chi_{z z}^{u}\left(H_{z}\right)\right\rangle}{\left\langle N_{0}(\mathbf{k}) \chi_{z z}^{u}\left(H_{z} \rightarrow 0\right)\right\rangle}=\frac{\chi_{z}}{\chi_{z 0}} \frac{1+4\left(\xi_{m} k_{F}\right)^{2}}{\frac{\chi_{z 0}}{\chi_{z}}+4\left(\xi_{m} k_{F}\right)^{2}} .
$$

Here the product $2 \gamma_{z z} k_{F}^{2} \chi_{z 0}=\left(\xi_{m} k_{F}\right)^{2}$ is expressed through the magnetic coherence length $\xi_{m}$ which near the zero temperature is of the order several interatomic distances.

In assumption $\left(\xi_{m} k_{F}\right)^{2} \gg 1$ one can rewrite the Eq. 40 as

$$
\left\langle N_{0}(\mathbf{k}) \chi_{z z}^{u}\left(H_{z}\right)\right\rangle \approx \frac{\chi_{z}\left(H_{z}\right)}{\chi_{z 0}}\left\langle N_{0}(\mathbf{k}) \chi_{z z}^{u}\left(H_{z} \rightarrow 0\right)\right\rangle .
$$

This very rough estimation presents the qualitative dependence of exponent in equation (37) from magnetic field. The longitudinal susceptibility drops with the augmentation of magnetic field parallel to the spontaneous magnetisation (see Fig 3 in the paper [37]) leading to the suppression of the temperature of transition to the superconducting state without including the orbital effect according Eq.(37).

Taking into account the orbital effect one can write the field dependence of critical temperature of transition to the superconducting state in the Ginzburg-Landau region

$$
T_{s c}^{o r b}(H)=T_{s c}(H)-\frac{H}{A T_{s c}(H)},
$$

where $A$ is a constant. Thus, the decreasing of $T_{s c}(H)$ with magnetic field causes not only faster drop but also the peculiar upward curvature in the critical temperature $T_{s c}^{o r b}(H)$ dependence from magnetic field in correspondence with the experimental data reported in 38 .

\section{B. Reentrant superconductivity in URhGe}

In field perpendicular to the spontaneous magnetisation the similar approach applied to the simplified single band model in weak coupling approximation yields (see Eq.(169) in the review [2]) the critical temperature

$$
\epsilon \exp \left(-\frac{T_{s c} \approx}{\left[\left\langle N_{0}(\mathbf{k}) \chi_{z z}^{u}\right\rangle \cos ^{2} \varphi+\left\langle N_{0}(\mathbf{k}) \chi_{y y}^{u}\right\rangle \sin ^{2} \varphi\right] I^{2}}\right)
$$

where $\tan \varphi=H_{y} / h$ and $h$ is the exchange field acting on the electron spins. This is the critical temperature of transition to the superconducting state without including the orbital effect.
The orbital effect suppresses the superconducting state and near the upper critical field at zero temperature

$$
H_{c 2 y}(T=0)=H_{0}=c T_{s c}^{2}
$$

the actual critical temperature is

$$
T_{s c}^{o r b}=a \sqrt{H_{0}-H_{y}},
$$

where $a \sqrt{c}$ is the numerical constant of the order of unity. This is the usual square root BCS dependence of the critical temperature from magnetic field in low temperature - high field region such that $T_{s c}^{o r b}\left(H_{y}=H_{0}\right)=0$. However, in the present case the magnitude $H_{0}$ itself is a function of the external field $H_{y}$. Let us look on its behaviour.

Similar to Eq. 41] we get

$$
\begin{aligned}
& \left\langle N_{0}(\mathbf{k}) \chi_{z z}^{u}\left(H_{y}\right)\right\rangle \cos ^{2} \varphi+\left\langle N_{0}(\mathbf{k}) \chi_{y y}^{u}\left(H_{y}\right)\right\rangle \sin ^{2} \varphi \approx \\
& \frac{\chi_{z}\left(H_{y}\right)}{\chi_{z 0}}\left\langle N_{0}(\mathbf{k}) \chi_{z z}^{u}\left(H_{y} \rightarrow 0\right)\right\rangle \cos ^{2} \varphi \\
& +\frac{\chi_{y}\left(H_{y}\right)}{\chi_{y 0}}\left\langle N_{0}(\mathbf{k}) \chi_{y y}^{u}\left(H_{y} \rightarrow 0\right)\right\rangle \sin ^{2} \varphi .
\end{aligned}
$$

Here, $\chi_{z}\left(H_{y}\right)$ and $\chi_{y}\left(H_{y}\right)$ are the $z$ and $y$ components of susceptibility in finite field $H_{y}$ and $\chi_{z 0}$ and $\chi_{y 0}$ are the corresponding susceptibilities at $H_{y} \rightarrow 0$. Unlike to the Eq. (41) the field dependence of the Eq. (46) is not so visible. One can note, however, the different field dependence of two summands in the Eq. (46).

(i) The susceptibility along $z$ direction $\chi_{z}\left(H_{y}\right)$ increases with magnetic field $H_{y}$ following to the decreasing of the Curie temperature according to Eq. (22). The growth of susceptibility along $\mathrm{z}$ direction at the approaching the field $H_{y}$ to $H_{c r}$ is confirmed by the field dependence of the NMR scattering rate $1 / T_{1}$ reported in [26, 27]. At the same time, the increase of $\chi_{z}\left(H_{y}\right)$ is limited by the decrease of $\cos ^{2} \varphi$. We do not know how fast it is because the magnitude of the exchange field is not known.

(ii) As the field approaches to $H_{c r}$ the low temperature susceptibility $\chi_{y}\left(H_{y}\right)$ has a high delta-function-like peak [7. with magnitude more than 10 times greater than it is at $H_{y} \rightarrow 0$. The factor $\sin ^{2} \varphi$ is also increased. This indicates that in URhGe the more important is the second term connected with the metamagnetic transition.

Thus, in vicinity of metamagnetic transition one can expect the increase of the critical temperature estimated without including the orbital effect according to Eq. 43. The radicand in the equation (45) after being negative in some field interval acquires the positive value as the field approaches to $H_{c r}$. The critical temperature Eq. (45) reaches maximum in vicinity of metamagnetic transition, see Fig2.

Similar arguments in favour of stimulation superconductivity near the metamagnetic transition in field parallel to $b$ axis can be applied to the discovered recently other superconducting compound $\mathrm{UTe}_{2}$ 39 41 isostructural with URhGe. However, in view of many particular 
properties of this material we leave this subject for future studies .

In the parent compound UCoGe the metamagnetic transition is absent (at least at $H_{y}<40 \mathrm{~T}$ ) [42. Hence, in this material the unusual temperature dependence of the upper critical field parallel to $b$ axis is probably mostly determined by the first term in the Eq. 46.

Near $H_{y}=H_{c r}$ at temperatures $T<T_{c r}$ the NMR spectrum is composed of two components indicating that the transition is of the first order accompanied by the phase separation [26]. Thus, in almost whole interval near $H_{c r}$ the superconductivity is developed in mixture of ferromagnetic state with polarisation along $z$ direction and the field polarised state with polarisation along $y$ direction.

\section{Upper critical field near metamagnetic transition in $\mathrm{UGe}_{2}$}

Peculiar example of superconductivity stimulation in vicinity of metamagnetic transition is realised in the other ferromagnetic compound $\mathrm{UGe}_{2}$. This material has orthorhombic structure with spontaneous magnetisation directed along a crystallographic direction. The magnetism in $\mathrm{UGe}_{2}$ has even more localised nature [2, 43, 44 than in related compounds URhGe and UCoGe. The superconductivity exists inside of ferromagnetic state in the pressure interval shown in Fig4. Inside of this interval at $P=P_{x}$ there is a metamagnetic transition from ferromagnetic state FM1 to ferromagnetic state FM2 characterised by the jump of spontaneous magnetisation from smaller to larger value 45. At a bit higher pressure $P=P_{x}+\delta P$ the transition from FM1 to FM 2 occurs in a finite magnetic field applied along the direction of spontaneous magnetisation. Near this transition in a finite field the magnetic susceptibility along the $a$-axis $\chi_{a}$ strongly increases. Hence, the critical temperature without including the orbital effect

$$
T_{s c}=\epsilon \exp \left(-\frac{1+\lambda}{\left\langle N_{0}(\mathbf{k}) \chi_{a}^{u}\right\rangle I^{2}}\right),
$$

growths up. As result the upper critical field in $a$ crystallographic direction measured at $P=P_{x}+\delta P$ acquires non-monotonic temperature dependence shown in Fig 5 [48, 49].

It worth to be noted that at pressures far from metamagnetic transition the upper critical field parallel to $a$ direction does not reveal an upward curvature [48, 49].
This important distinction from the upper critical field behaviour in UCoGe considered in Section IIIA is related to the difference of susceptibility dependence from magnetic field along spontaneous magnetisation in these two materials. Whereas in UCoGe the susceptibility $\chi_{c}$ along $c$-axis is strongly field dependent 37, in $\mathrm{UGe}_{2}$ the susceptibility $\chi_{a}$ along $a$-axis is practically field independent [45, 50].

\section{CONCLUSION}

We have demonstrated that in the orthorhombic ferromagnet URhGe the ferromagnetic ordering along $c$ axis suppressed in process of increase of magnetization in the perpendicular $b$-direction induced by the external magnetic field. This process is accelerated by the tendency to the metamagnetic transition which occurs at $H_{y}=H_{c r}=12 \mathrm{~T}$. The transition of the first order is accompanied by the suppression of the ferromagnetic state with polarisation along $c$-axis and the arising of magnetic state polarised along $b$-xis. The line of first order phase transition is finished at the critical end point with temperature $T=T_{c r}=4 \mathrm{~K}$.

The uniaxial stress along $b$-axis causing moderate suppression of the Curie temperature in the absence of magnetic field accelerates the Curie temperature drop in finite magnetic field $H_{y}$ and quite effectively decreases the critical field of metamagnetic transition. As result, the superconducting state recovers itself in much smaller field and can even merged with superconducting state in the small fields region.

The superconducting pairing is determined by the exchange interaction between the conduction electrons and the magnetic moments localised on uranium atoms.

In UCoGe the upward curvature of the upper critical field along c-axis is mostly determined by the longitudinal magnetic susceptibility decrease along with the magnetisation saturation.

In URhGe the superconducting state suppressed in field $H_{y} \approx 2 \mathrm{~T}$ is recovered in fields interval $(9-13) \mathrm{T}$ near the critical field. This phenomenon is related to the strong increase of the pairing interaction caused mostly by the strong augmentation of the magnetic susceptibility along $b$-direction in vicinity of the metamagnetic transition.

The nonmonotoneous behaviour of the upper critical field in UGe2 is explained by the strong increase of longitudinal magnetic susceptibility at the metamagnetic transition from FM1 to FM2.
[1] D.Aoki, K.Ishida and J.Flouquet, J. Phys. Soc. Jpn. 88, 022001 (2019).

[2] V.P.Mineev, Usp. Fiz. Nauk 187, 129 (2017) [Phys.-Usp. 60, 121 (2017).

[3] Levy F, Sheikin I, Grenier B, Huxley A D Science 309
$1343(2005)$

[4] F. Hardy, A.D. Huxley, Phys. Rev. Lett. 94, 247006 (2005).

[5] V.P.Mineev, Phys. Rev. B 91014506 (2015).

[6] F.Hardy, D.Aoki, C.Meingast, P.Schweiss, P.Burger, H. 
v. Löhneysen, and J.Flouquet, Phys.Rev.B 83, 195107 (2011).

[7] S.Nakamura, T.Sakakibara, Y.Shimizu, S.Kittaka, Y.Kono, Y.Haga, J.Pospisil, and E.Yamamoto, Phys. Recv. B 96, 094411 (2017).

[8] Y. Aoki, T.D. Matsuda, H. Sugawara, H. Sato, H. Ohkuni, R. Settai, Y. Onuki, E. Yamamot, Y. Haga, A.V. Andreev, V. Sechovsky, L. Havela, H. Ikeda, K. Miyake, Journ.Mag.Magn.Mat. 177-181,271 (1998).

[9] D.Aoki, T.Combier, V.Taufour, T.D.Matsuda, G.Knebel, H.Kotegawa, and J.Flouquet, J.Phys. Soc. Jpn. 80, 094711 (2011).

[10] A.B.Shick, Phys.Rev. B 65, 180509(R) (2002).

[11] R.Z.Levitin, A.S.Markosyan, Usp. Fiz. Nauk 155, 623 (1988) [Phys.-Usp. 31, 730 (1988).

[12] F.Wilhelm, J.P.Sanchez, J.-P.Brison, D.Aoki, A.B.Shick, and A.Rogalev, Phys.Rev. 95, 235147 (2017).

[13] F. Hardy, A. Huxley, J. Flouquet, B. Salce, G. Knebel, D. Braithwaite, D. Aoki, M. Uhlarz, C. Pfleiderer, Physica B 359-361, 1111 (2005).

[14] A.Miyake, D.Aoki, and J. Flouquet, J.Phys. Soc. Jpn. 78, 063703 (2009).

[15] D.Braithwaite, D.Aoki, J.-P.Brison, J.Flouquet, G.Knebel, A.Nakamura, and A.Pourret, Phys. Rev. Lett. 120, 037001 (2018).

[16] V.P.Mineev, Phys. Rev. B 95, 104501 (2017).

[17] L.D.Landau and E.M.Lifshitz, Statistical Physics, Course of Theoretical Physics Vol V. Oxford: Butterworth-Heinemann,1995).

[18] A.Miyake, D.Aoki, and J. Flouquet, J.Phys. Soc. Jpn. 77, 094709 (2008).

[19] A. Gourgout, A. Pourret, G. Knebel,D. Aoki,G. Seyfarth,and J. Flouquet, Phys.Rev.Lett. 117, 046401 (2016).

[20] R.W.Keyes, J. Phys. Chem. Solids 6, 1 (1958).

[21] V.F.Gantmakher, I.B.Levinson, Zh. Eksp. Teor. Fiz. 74, 261 (1978) [Sov. Phys. JETP 47133 (1978)].

[22] J.Appel and A.W.Overhauser, Phys.Rev.B 18, 7581978.

[23] S.S.Murzin, S.I.Dorozhkin, A.C.Gossard, Pis'ma Zh. Eksp. Teor.Fiz. 67, 101 (1998) [JETP Letters 67, 113 (1998)].

[24] H.K.Pal, V.I.Yudson, and D.L.Maslov, Lith.J.Phys. 52, 142 (2012).

[25] K.Prokes, T.Tahara, Y.Echizen,T.Takabatake,T.Fujita, I.H.Hagmusa, J.C.P.Klaasse, E.Brück, F.R.deBoer, M.Divis, V.Sechovsky, Physica B 311, 220 (2002).

[26] H.Kotegawa, K.Fukumoto, T.Toyama, H.Tou, H.Harima, A.Harada, Y.Kitaoka, Y.Haga, E.Yamamoto, Y.Onuki, K.M.Itoh, and E.E.Haller, J.Phys. Soc. Jpn. 84, 054710 (2015).

[27] Y. Tokunaga, D.Aoki, H.Mayaffre, S. Krämer, M.-H. Julien,C. Berthier, M. Horvati?, H. Sakai, S. Kambe, and S. Araki, Phys. Rev. Lett. 114, 216401 (2015).

[28] W.L.McMillan, Phys.Rev.167, 331 (1968).
[29] D. Fay and J. Appel: Phys. Rev. B 22 (1980) 3173.

[30] W. F. Brinkman and S. Engelsberg, Phys. Rev. 169, 417 (1968).

[31] Yu.Sherkunov, A.V. Chubukov, and J.J.Betouras, Phys.Rev.Lett. 121, 097001 (2018).

[32] E.A.Yelland, J.M.Barraclough, W.Wang, K.V. Kamenev, and A.D. Huxley, Nat. Phys. 7, 890 (2011).

[33] M.Taupin, J.P.Sanchez, J.-P.Brison, D.Aoki, G.Lapertot, F.Wilhelm, and A.Rogalev, Phys.Rev. 92, 035124 (2015).

[34] R.Hattori, and H.Tsunetsugu, Phys. Rev. B 87, 064501 (2013).

[35] N.Karchev, Phys.Rev.B 67, 054416, (2003).

[36] V.P.Mineev, Annals of Physics (NY), to be published (2020).

[37] N.Y.Huy, D.E.de Nijs, Y.K.Huang, and A.de Visser, Phys.Rev.Lett. 100, 077002 (2008).

[38] B.Wu, G.Bastien, M.Taupin, C.Paulsen, L.Howald, D.Aoki and J.-P. Brison, Nature Comm. 8, 14480 (2017).

[39] S.Ran, I-Lin Liu, YunSuk Eo, D.J.Campbell, P.M.Neves, W.T.Fuhrman, S.R.Saha, C.Eckberg, H.Kim, D.Graf, F.Balakirev, J.Singleton, J.Paglione and N.Butch, Nature Physics 15,1250 (2019).

[40] G.Knebel, W.Knafo, A.Pourret, Qun Niu, M.Valiska, D.Braithwaite, G.Lapertot, M.Nardone, A.Zitouni, S.Mishra, I.Sheikin, G.Seyfarth, J.-P.Brison, D.Aoki, J.Flouquet, J. Phys. Soc. Jpn 88, 063707 (2019).

[41] Q.Niu, G.Knebel, D.Braithwaite, D.Aoki, G.Lapertot, M.Valiska, G.Seyfarth, W.Knafo, T.Helm, J.-P.Brison, J.Flouquet, and A.Pourret, arXiv:2003.08986 [cond-mat] (2020).

[42] W.Knafo, T.D.Matsuda, D.Aoki, F.Hardy, G.W.Scheerer, G.Ballon, M.Nardone, A.Zitouni, C.Meingast and J.Flouquet, Phys. Rev. B 86, 184416 (2012).

[43] R.Troc, Z. Gajek, and A.Pikul, Phys. Rev. B 86, 224403 (2012).

[44] V.P.Mineev, Phys. Rev. B 88224408 (2013).

[45] C.Pfleiderer and A.D.Huxley, Phys. Rev. Lett. 89, 147005 (2002).

[46] F. Hardy, C. Meingast, V. Taufour, J. Flouquet, H. v. Löhneysen, R. A. Fisher, N. E. Phillips, A. Huxley, and J. C. Lashley, Phys.Rev.B 80174521 (2009).

[47] V. Taufour, D. Aoki, G. Knebel, and J. Flouquet, Phys. Rev. Lett. 105, 217201 (2010).

[48] A.Huxley, I.Sheikin, E.Ressouche, N.Kernavanois, D.Braithwaite, R.Calemczuk, and J.Flouquet, Phys. Rev. B 63, 144519 (2001).

[49] I.Sheikin, A. Huxley, D. Braithwaite, J. P. Brison, S. Watanabe, K. Miyake, and J. Flouquet, Phys.Rev. B 64, 220503(R) (2001).

[50] N.Tateiwa, Y.Haga, and E.Yamamoto, Phys.Rev.Lett. 121, 237001 (2018). 


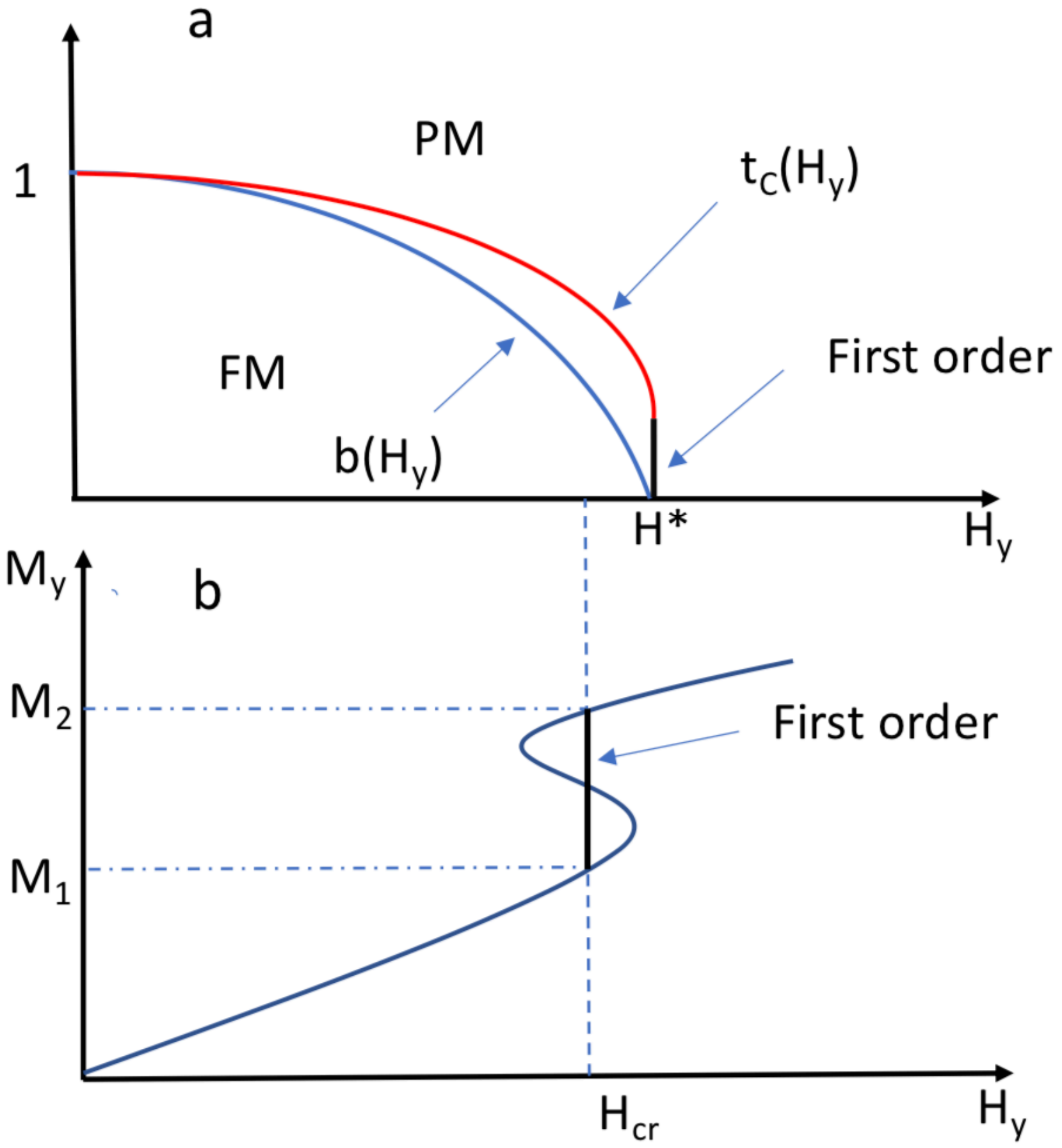

FIG. 1: (Color online) a) Schematic behaviour of the normalised Curie temperature $t_{c}\left(H_{y}\right)=\frac{T_{c}\left(H_{y}\right)}{T_{c 0}}$ and coefficient $b\left(H_{y}\right)=\frac{\tilde{\beta}_{z}}{\beta_{z}}$. FM and PM stand for ferromagnetic and paramagnetic phases. b) Schematic dependence $M_{y}\left(H_{y}\right)$ at $T<T_{c r}$ and $H_{c r}<H^{\star}$. 


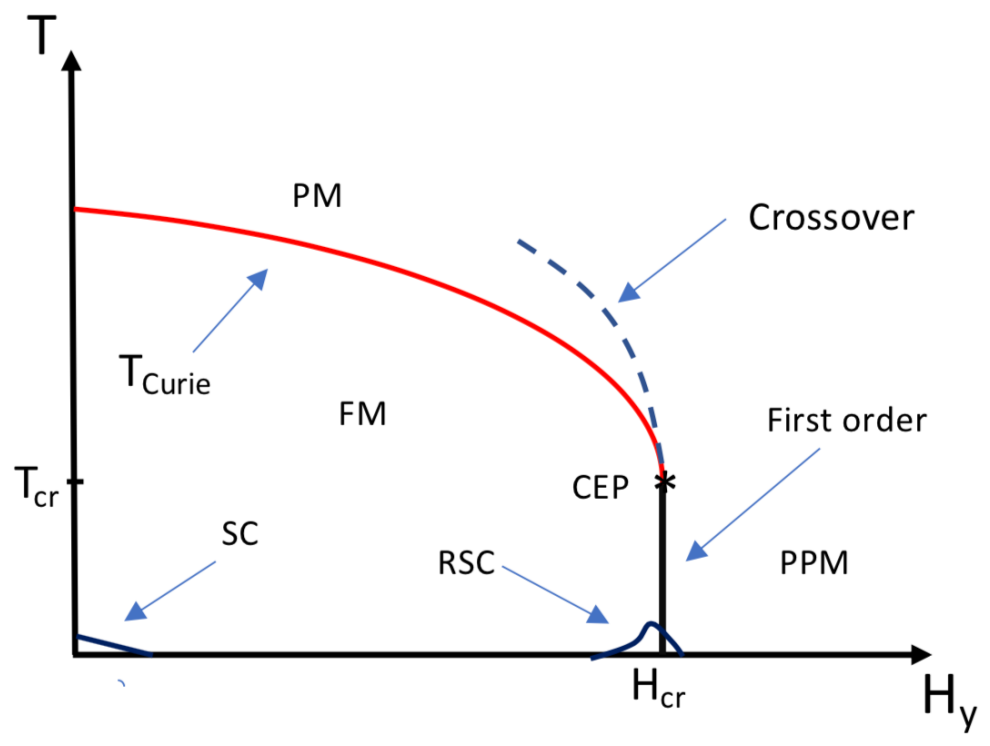

FIG. 2: (Color online) Phase diagram UCoGe in magnetic field parallel to $b$-crystallographic direction. PM, FM, PPM denote paramagnetic, ferromagnetic and polarised paramagnetic phases. CEP is the critical end point. SC and RSC are the superconducting and reentrant superconducting states. 


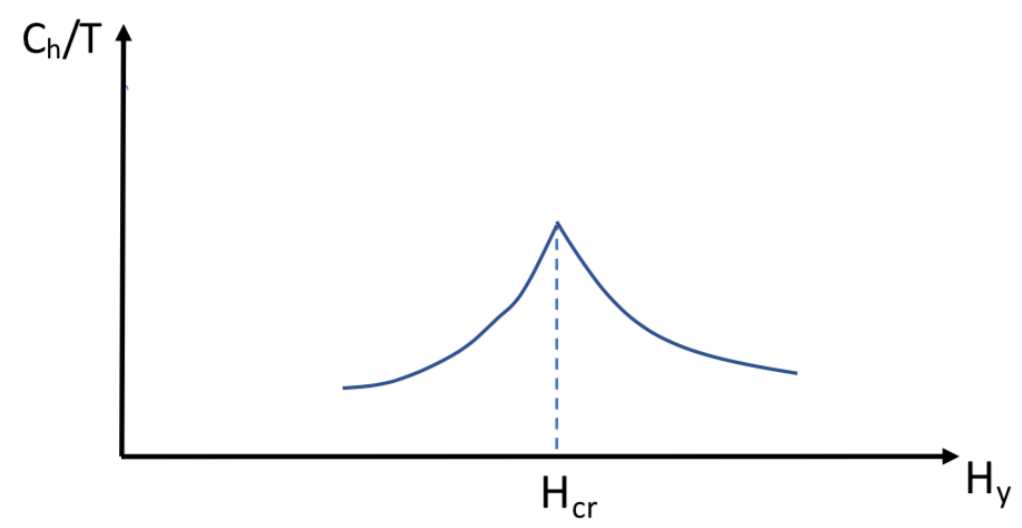

FIG. 3: (Color online) Schematic behaviour $C_{h} / T$ (See the main text). 


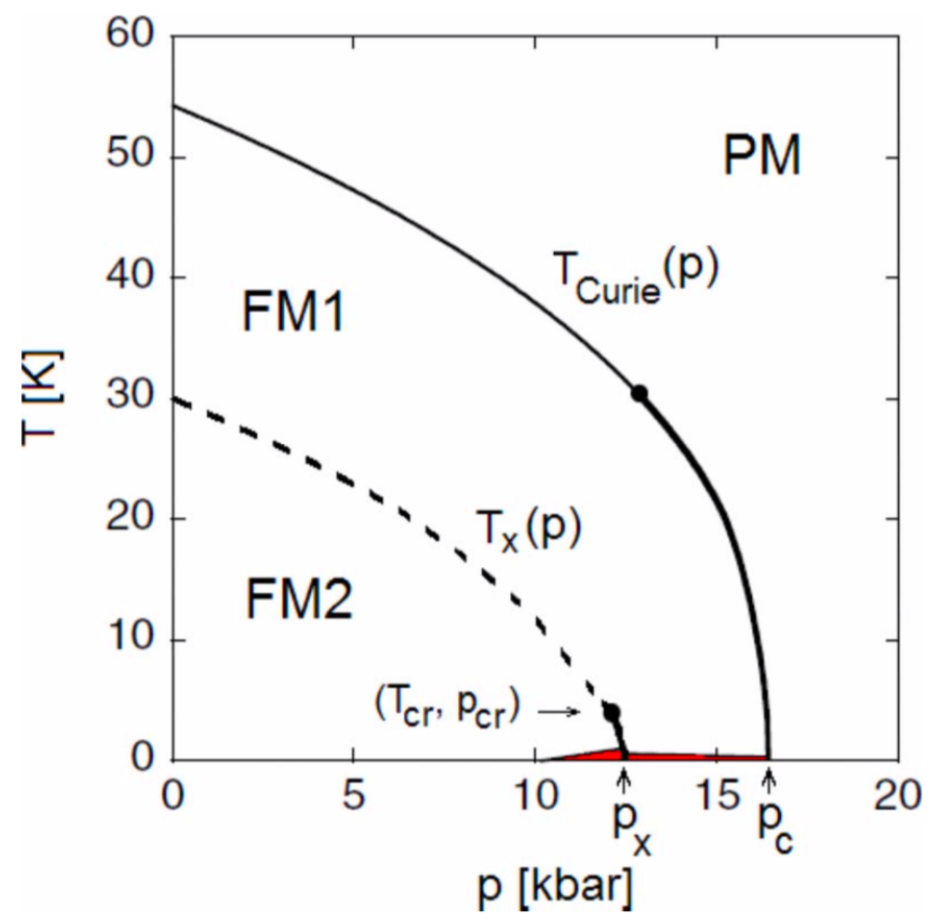

FIG. 4: (Color online) The schematic $P, T$ phase diagram of $\mathrm{UGe}_{2}$ 45, 46. Thick lines represent first-order transitions and thin lines denote second-order transitions. The dashed line indicates a crossover while the dots mark the positions of critical points. The superconducting region is represented in red area at bottom. 


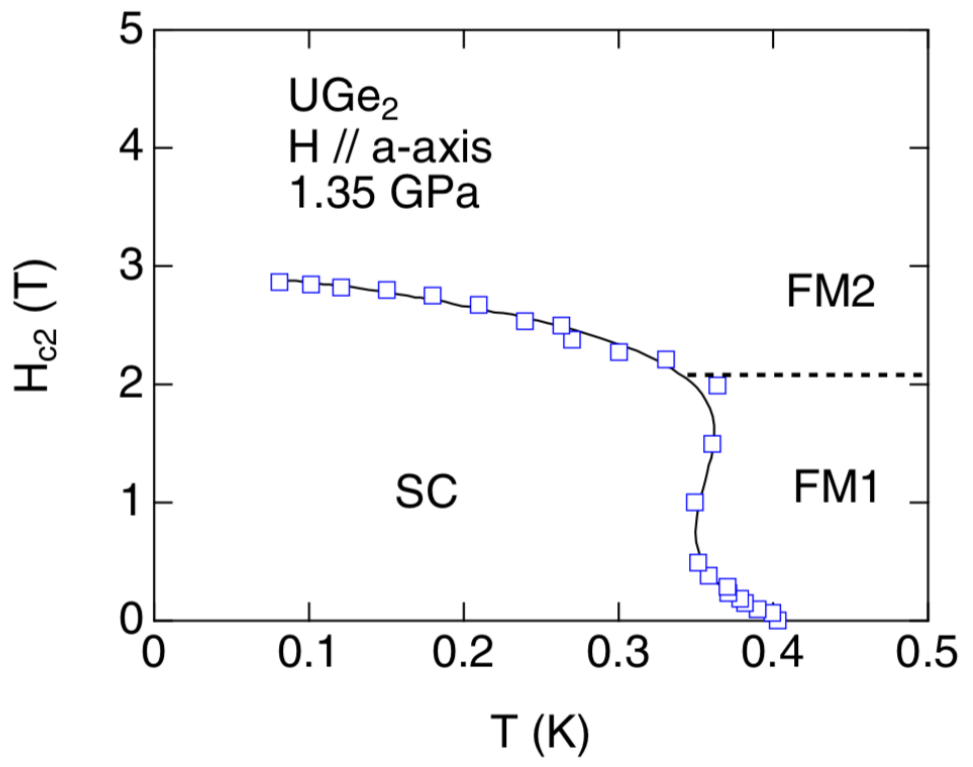

FIG. 5: (Color online) Temperature dependence of $H_{c 2}$ for field parallel to $a$-axis in $\mathrm{UGe}_{2}$ at $1.35 \mathrm{GPa}$, which is just above $P_{x}$. The metamagnetic transition is detected at $H_{x}$ between FM1 and FM2 48, 49]. 